\title{
THE EFFECT OF TRANSTOOTH CURING TECHNIQUE USING DIFFERENT LED LIGHT INTENSITIES ON MICROHARDNESS AND DEPTH OF CURE OF NANOFILLED RESIN COMPOSITE RESTORATIONS
}

\author{
Shereen Hafez
}

\begin{abstract}
Aim: To evaluate the effect of different LED light intensities on microharness and depth of cure of nanofilled resin composite restorations using transtooth illumination technique with different thickness of buccal wall.

Materials and methods: The materials used for this study was nanofilled resin composite material (Filtex Z350XT, 3M, ESPE) shade A3 with its corresponding bonding system (single bond universal, 3M, ESPE). A total of 10 sound extracted human maxillary premolar teeth were used in this study. The teeth were divided into 2 main groups of 5 teeth each according to curing light intensities; either standard LED curing mode $\left(800 \mathrm{~mW} / \mathrm{cm}^{2}\right)$ for $30 \mathrm{sec}$ or high intensity LED curing mode $\left(1400 \mathrm{~mW} / \mathrm{cm}^{2}\right)$ for $10 \mathrm{sec}$. In each tooth, 2 cavity preparations were performed; moderate cavity preparation width (1/3 intercuspal distance) in the mesial aspect of each tooth and wide cavity preparation (1/2 intercuspal distance) in the distal aspect. All specimens were light cured using transtooth curing technique from the buccal and lingual surfaces. Each tooth was sectioned in a mesio-distal direction into 2 equal halves. Surface Micro-hardness of the specimens was determined. The mean microhardness values and hardness ratio $\%$ of the specimens were calculated. One way analysis of variance ANOVA, Tukey's post hoc and independent $t$ tests were used to compare between more than 2 groups. The significance level was set at $\mathrm{p} \leq 0.05$.
\end{abstract}

Results: The highest mean value for B/T ratio was recorded in Post. High I/3 ICD, whereas the lowest mean was recorded in Post.conv. I/3 ICD. ANOVA test revealed a significance difference between all groups $(\mathrm{p}<0.0001)$. Tukey's post hoc test revealed no significant difference between the post high $1 / 2$ ICD group and the post conv. 1/3 ICD group. Comparisons within the same groups revealed a significance difference within the post high groups, and within the post Conv. Groups $(\mathrm{p}=0.0007, \mathrm{p}=0.012$ respectively. Moreover, there was a significant difference between the $1 / 3$ subgroups $(\mathrm{p}<0.0001)$. However, the difference between the $1 / 2$ ICD subgroups was not statistically significant $(\mathrm{p}=0.1174)$.

Conclusions: Transtooth curing techniques is greatly affected by cavity width. Light intensity is critical factor that should be considered when performing transtooth curing techniques. High LED light intensity successfully able to perform optimum polymerization for resin composite in both cavity width (moderate and wide).

KEY WORDS: High LED light intensiy, transtooth curing, depth of cure, hardness ratio, nanofilled composite

* Lecturer of Conservative Dentistry, Faculty of Dentistry, Cairo University 


\section{INTRODUCTION}

In the last few years, resin composite materials had become universal restoratives due to increase patient esthetic demands. Every effort had been directed to overcome one of the major problem encountered with these materials known as polymerization shrinkage and stresses with their consequences in marginal gap formation, microleakage, debonding, secondary caries and postoperative sensitivity. ${ }^{1-3}$ Several modifications had been introduced in resin composite materials and their techniques of applications, never the less, type of curing light, curing modes and exposure time. ${ }^{4,5}$

The incremental placement of resin composite material has been routinely used by the clinicians associated with photo-activation from occlusal direction for adequate light penetration. However, the benefit of using an incremental technique for reducing shrinkage stresses might be questionable, as it might comprise the possibility of trapping voids or contamination between layers and the increased time required to place the restoration. ${ }^{5,6}$ The direction of shrinkage is another important factor in controlling shrinkage patterns in restorations as it was supposed that resin composite shrinks toward the source of light. However, some authors demonstrated that the direction of shrinkage is determined predominantly by the presence or absence of a bond. ${ }^{7-9}$

Transtooth curing has been introduced as guided polymerization technique for restoration of prepared class II cavity to provide better adaptation of resin composite restoration. However, the curing efficiency of these restoration is very questionable as the as light passes through buccal wall, it is greatly reduced due to light scattering resulting in decrease in the effectiveness of cure at the bottom surface of resin composite restoration. ${ }^{10-12}$ Researchers suggested that a transtooth illumination technique involving light curing from buccal and lingual directions of a bulk-placed composite could be beneficial while avoiding the disadvantages of incremental techniques. ${ }^{6}$ The main concern of the current study is whether the composite cures fully enough in the deeper portions to create a material that has acceptable physical and biocompatible properties. Furthermore, does the amount of tooth structure at the buccal or lingual wall of the prepared cavity and the intensity of light curing will affect the depth of cure of resin composite materials. Hence, this study is carried out to evaluate the effect of different LED light intensities on microharness and depth of cure of nanofilled resin composite restorations using transtooth illumination technique with different thickness of buccal wall.

\section{MATERIALS AND METHODS}

The materials used for this study was nanofilled resin composite material (Filtex Z350XT, 3M, ESPE, St Paul,MN,USA Batch\#N731284) shade A3with its corresponding bonding system (single bond universal, 3M, ESPE). The resin composite contains, bis-GMA, UDMA, TEGDMA, and bis-EMA resins according the manufacturer. PEGDMA has been substituted for a portion of the TEGDMA resin to moderate the shrinkage. The fillers are a combination of non-agglomerated/nonaggregated $20 \mathrm{~nm}$ silica filler, non-agglomerated/ non-aggregated 4 to $11 \mathrm{~nm}$ zirconia filler, and aggregated zirconia/silica cluster filler (comprised of $20 \mathrm{~nm}$ silica and 4 to $11 \mathrm{~nm}$ zirconia particles). The inorganic filler loading is about $72.5 \%$ by weight (55.6\% by volume). For photo-activation, LED curing unit (Dr's light AT, Good doctors co.ltd. korea) with two different light intensities were used in this study.

\section{Sample preparation:}

A total of 10 sound extracted human maxillary premolar teeth were used in this study. The selected teeth had an average occluso-gingival height of teeth $4.3 \mathrm{~mm}$ and their average bucco-lingual dimension was 8.6-10.5 $\mathrm{mm}$ that was checked using 
a digital caliper (Ruifeng Foreign, China). Teeth with a minimum occluso-gingival height of were selected. The teeth were divided into 2 main groups of 5 teeth each according to curing light intensities; either standard LED curing mode $\left(800 \mathrm{~mW} / \mathrm{cm}^{2}\right)$ for $30 \mathrm{sec}$ or high intensity LED curing mode (1400 $\mathrm{mW} / \mathrm{cm}^{2}$ ) for $10 \mathrm{sec}$. In each tooth, 2 cavity preparations were performed; moderate cavity preparation width $(1 / 3$ intercuspal distance) in the mesial aspect of each tooth and wide cavity preparation ( $1 / 2$ intercuspal distance) in the distal aspect. Thus, a total of 20 cavity preparations were performed. All specimens were light cured using transtooth curing technique from the buccal and lingual surfaces.

Two standardized, compound class II cavity preparations were prepared on the proximal surfaces of all teeth using no. 245 carbide bur (Healthico, USA) in a high speed hand-piece (PANA MAX, NSK, Japan) under copious amount of water. The carbide bur was changed every 10 preparations. Cavity preparation dimensions were then measured using a digital caliper and the outline was drawn. The cavity preparation dimensions were approximately standardized to $4 \mathrm{~mm}$ occluso-gingival height and $1.5 \mathrm{~mm}$ axial wall depth. Regarding the width, the mesial cavity preparation was standardized to 3 $\mathrm{mm}$ bucco-lingual width representing the moderate cavity width, while the distal cavity preparation was $4 \mathrm{~mm}$ for wide cavity preparation.

\section{Filling and curing technique:}

Each tooth with mesial and distal prepared cavities was placed in dental study model (El Banna, Egypt) with proper mesial and distal contact with adjacent ivory teeth. A pre-contoured Sectional matrix, standard kit (Palodent Sectional Matrix System, Dentsply, USA) was used. Two standard size matrices with BiTine round rings were placed mesially and distally with proper wooden wedge size. All prepared cavities were restored using nanofilled resin composite material (Filtex Z350XT, 3M, ESPE). The same bonding protocol was performed, according to manufacturer's instructions, for all groups. The adhesive system (single bond universal,3M,ESPE) was applied using disposible microbrush, agitated for $10 \mathrm{sec}$ and then the adhesive was thinned with gentle air stream using light pressure for 5 seconds and polymerized using a LED curing unit $\left(800 \mathrm{~mW} / \mathrm{cm}^{2}\right)$ for 20 seconds, with perpendicular angulation at zero distance. The resin composite was placed incremently in both cavities using a gold-plated composite applicator (Miltax, Germany). The resin composite material was then light cured using LED light curing unit according to the previously described testing groups using different predetermined light intensities. Before each curing cycle, the light irradiance was checked using the built-in radiometer. For Transtooth curing technique, before light curing, the matrix ring retainers were removed and the band ends were slightly displaced such that the curing tip is in direct contact with the tooth and one light curing cycle was applied from buccal and lingual transtooth direction.

\section{Micro-hardness testing for depth of cure assessment}

Each tooth was sectioned in a mesio-distal direction into 2 equal halves. Then each tooth half was poured in orthodontic resin leaving the cut surface uncovered. Prior to the Micro-hardness measurement, the previous specimens were longitudinally polished using a sequence of 8001200-4000 grit silicon carbide paper and alumina polishing paste $(1 \mu \mathrm{m})$. Surface Micro-hardness of the specimens was determined using Digital Display Vickers Micro-hardness Tester (Model HVS-50, Laizhou Huayin Testing Instrument Co., Ltd. China) with a Vickers diamond indenter and a $20 \mathrm{X}$ objective lens. A load of $100 \mathrm{~g}$ was applied to the surface of the specimens for 20 seconds. Three indentations, which were equally placed over a circle and not closer than $0.5 \mathrm{~mm}$ to the adjacent indentations, were made on the surface of each specimen. The diagonals length of the indentations 
were measured by built in scaled microscope and Vickers values were converted into micro-hardness values.

\section{Micro-hardness calculation:}

Micro-hardness was obtained using the following equation:

$$
H V=1.854 P / d^{2}
$$

where, $\mathbf{H V}$ is Vickers hardness in $\mathrm{Kgf} / \mathrm{mm}^{2}, \mathbf{P}$ is the load in Kgf and $\mathbf{d}$ is the length of the diagonals in $\mathrm{mm}$.

Five VK readings were recorded for each sample surface (top and bottom). For a given specimen, the five hardness values for each surface were averaged and reported as a single value. The mean microhardness values and hardness ratio $\%$ of the specimens were calculated and tabulated using the formula:

Hardness ratio $\%=\mathrm{VK}$ of bottom surface $/ \mathrm{VK}$ of top surface X 100

\section{Statistical analysis:}

Values were presented as mean and standard deviation (SD) values. Data were explored for normality using Kolmogorov-Smirnov test of normality. The results of Kolmogorov-Smirnov test indicated that data were normally distributed (parametric data), so one way analysis of variance ANOVA, Tukey's post hoc and independent $t$ tests were used to compare between more than 2 groups. The significance level was set at $\mathrm{p} \leq 0.05$. Statistical analysis was performed with SPSS 18.0 (Statistical Package for Scientific Studies, SPSS, Inc., Chicago, IL, USA) for Windows.

\section{RESULTS}

The highest mean value for $\mathrm{B} / \mathrm{T}$ ratio was recorded in Post. High I/3 ICD, whereas the lowest mean was recorded in Post.conv. I/3 ICD. ANOVA test revealed a significance difference between all groups $(\mathrm{p}<0.0001)$. Tukey's post hoc test revealed no significant difference between the post high $1 / 2$ ICD group and the post conv. 1/3 ICD group. Comparisons within the same groups revealed a significance difference within the post high groups, and within the post Conv. Groups ( $\mathrm{p}=0.0007$, $\mathrm{p}=0.012$ respectively), (Table 1, Fig.1). Moreover, there was a significant difference between the $1 / 3$ subgroups $(\mathrm{p}<0.0001)$. However, the difference between the $1 / 2$ ICD subgroups was not statistically significant $(\mathrm{p}=0.1174)$, (Table 2$)$.

TABLE (1) Comparison of B/T ratio in high and Conv. Groups (ANOVA and Tukey's post hoc test)

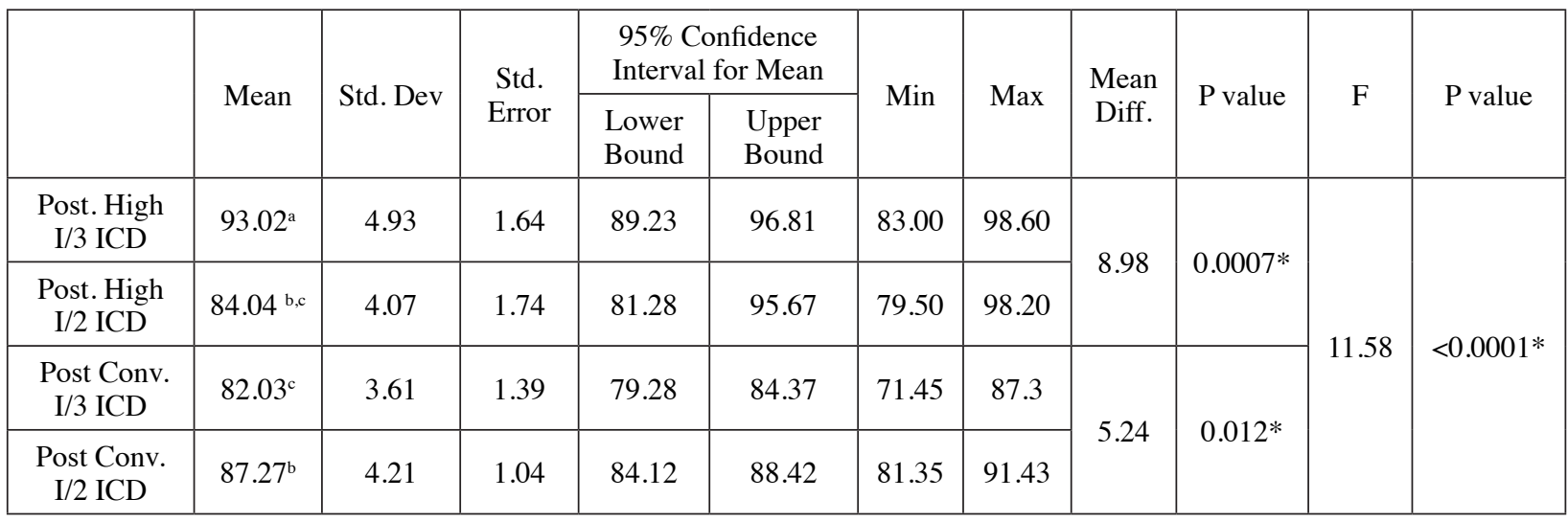

Significance level $p<0.05$, * non-significant

Tukey's post hoc test: means sharing the same superscript letter are not significantly different 
Table (2) Comparison of $\mathrm{B} / \mathrm{T}$ ratio within the same ICD ( $\mathrm{t}$ test)

\begin{tabular}{|c|c|c|c|c|}
\hline \multirow{2}{*}{} & \multicolumn{2}{|c|}{ I/3 ICD } & \multicolumn{2}{c|}{ I/2 ICD } \\
\cline { 2 - 5 } & Post. High & Post Conv & Post. High & Post Conv \\
\hline Mean \pm SD & $93.02 \pm 4.93$ & $82.03 \pm 3.61$ & $84.04 \pm 4.07$ & $87.27 \pm 4.21$ \\
\hline $\begin{array}{c}\text { Mean } \\
\text { difference }\end{array}$ & \multicolumn{2}{|c|}{10.99} & \multicolumn{2}{|c|}{3.23} \\
\hline $\begin{array}{c}95 \% \\
\text { confidence } \\
\text { interval }\end{array}$ & \multicolumn{2}{|c|}{6.6722 to 15.3078} & 0.9078 to 7.3678 \\
\hline P & \multicolumn{2}{|c|}{$<0.0001 *$} & \multicolumn{2}{|c|}{$0.1174^{\text {ns }}$} \\
\hline
\end{tabular}

Significance level $p<0.05, \quad *$ significant, $n s=$ non-significant

\section{DISCUSSION}

The physical and mechanical properties of resin composites are strongly influenced by the degree of conversion, defined as the percentage of reacted aliphatic $\mathrm{C}=\mathrm{C}$ bonds from the dimethacrylate monomers present in their polymeric matrices. Thus, the extent of this response plays a crucial role in the clinical performance of resin composite restorations ${ }^{(15)}$. While, the "depth of cure" usually refers to the thickness of a resin based composite that is adequately cured, and it is always limited by light absorption and scatter within the material, which are influenced by numerous factors. ${ }^{(16)}$

Little published information exists about the effectiveness of transtooth-illumination techniques on the microhardness and depth of cure of resin composite. This study was carried out to evaluate the effect of different LED light intensities on microharness and depth of cure of nanofilled resin composite restorations using transtooth illumination technique with different thickness of buccal wall. In this study, every effort was exerted to standardize the methodology and simulate the clinical conditions. A standardized box-shaped preparation was made on mesial and distal of each tooth for the moderate width (1/3 ICD) and wide (1/2 ICD) cavity preparations. In addition, a standardized cavity

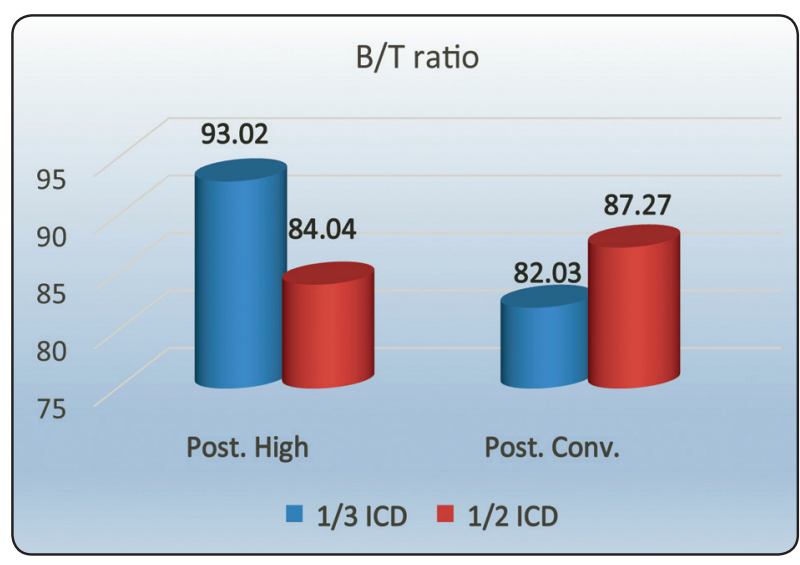

Fig.(1) Column chart showing mean $\mathrm{B} / \mathrm{T}$ ratio in high and Conv. Groups

preparation dimension would result in a standard amount of composite restoration to be polymerized.

Since the depth of cure is an important tool to estimate the properties of composite resin restorations ${ }^{(17)}$, different methods are available to assess the depth of cure of resin composite restoration. The surface hardness measurements, whether Vickers or Knoop indenters, is one of the most widely used method for indirect determination of the depth of cure of resin composites. A good correlation was found between increasing hardness and increasing depth of cure ${ }^{(18)}$. Thus, in this study the depth of cure was assessed using Digital Display Vickers Microhardness Tester, since it is easy to apply and the data obtained are reliable (17). Moreover, in this study Vickers microhardness number (VHN) was determined on the top, middle and the bottom surfaces for each specimen since the light intensity is attenuated when passing through the composite due to light scattering effect of filler/matrix refractive index mismatch ${ }^{(18)}$. As a consequence, the bottom surface is more critically affected by the light intensity, so it is considered as a good surface to estimate the effectiveness of cure of composite ${ }^{(17)}$. Moreover, measuring the microhardness on the middle area of restoration was essential to infer the effect of transtooth curing. The hardness value obtained at each surface was 
the mean of 4 indentations ( 2 on each half) to reduce variability between hardness values. Thus, assessment of bottom/top surface hardness percent is a valuable indicator of the thoroughness of cure of a sample. Ideally, the degree of polymerization of the composite should be the same throughout its depth and the hardness percentage should be very close or equal to hundred. However, there is a general consensus in the literature that a value of $80 \%$ bottom/top percentage is considered the minimally acceptable value for light cured resincomposites ${ }^{(11,16-19)}$.

In the current study, Microhardness ratio \% results of all tested groups was above $82 \%$ that could be considered the minimally acceptable value. This may denote that the transtooth curing techniques alone not in conjuction with occlusal curing technique could provide acceptable degree of conversion and depth of cu re for nanofilled resin composite materials. The superior performance of the transtooth-illumination technique in this study could be a result of the ability of light to penetrate enamel walls, or it simply could arise from the fact that the amount of light energy applied with this technique was higher. ${ }^{(11)}$

However, detailed results revealed that the highest mean value for Microhardness ratio \% was recorded in Post. High I/3 ICD, whereas the lowest mean was recorded in Post.conv. I/3 ICD denoting that cavity width as well as the LED light intensity greatly affect the depth of cure of resin composite materials. Comparisons within the same groups revealed a significance difference within the post high groups, and within the post Conv. Groups (Table 1, Fig.1). This might emphasize the importance of cavity width and cavity wall thickness as a critical factor to be considered during performing transtooth curing technique. It should be pointed out that in conventional LED intensity, moderate cavity width (1/3 ICD) recorded less microhardneess ratio\% compared to wide cavity (1/2ICD). This might necessitate the presence of occlusal curing techniques in conjunction with transtooth curing in moderate sized cavity to ensure optimum polymerization as the light intensity might be diminished due to thick cavity wall. However, this conventional curing LED intensity was efficient in wide cavity as the cavity wall was less, so permit sufficient amount of light to pass through and provide adequate polymerization for resin composite. Moreover, the difference between the $1 / 2$ ICD subgroups was not statistically significant. Denoting that regardless the curing light intensity used (conventional or high LED) in wide cavity, the cavity wall thickness will permit sufficient light to provide adequate polymerization for resin composite.

The most surprising result was that the high intensity light curing mode was successfully able to perform optimum polymerization for resin composite in both cavity width (moderate and wide). Although there is some suspicion about the effectiveness of techniques that involve curing through tooth structure, investigators reported that light could penetrate enamel and dentin walls and cure the inner portion of a restoration with no difference compared with the incremental technique, provided that the composite was cured from the buccal, lingual and occlusal directions. ${ }^{(15,16)}$ Thus, the results of the current study suggest that clinicians should be more concerned about a thorough cure of a restoration.

\section{CONCLUSIONS}

Under the limitation of the current study the following conclusion could be derived:

1. Transtooth curing techniques is greatly affected by cavity width.

2. Light intensity is critical factor that should be considered when performing transtooth curing techniques.

3. High LED light intensity successfully able to perform optimum polymerization for resin composite in both cavity width (moderate and wide). 


\section{REFERENCES}

1. RG. Craig, R. Sakaguchi, Resin composite restorative materials. Restorative dental materials. 12th ed. Mosby; 2006.189-212.

2. S. Bayne, G. Thompson, D. Taylor. Dental materials. In: Roberson TM, Heyman HO, Swift EJ, editors. Sturdevant's art \& science of operative dentistry. 4th ed. Mosby; 2002. p.135-234.

3. Letzel H. Survival rates and reasons for failure of posterior composite restorations in multicentre clinical trial. J Dent 1989; 17(suppl 1):S10-S17.

4. Loguercio AD, Reis A, Schroeder M, Balducci I, Versluis A, Ballester RY. Polymerization shrinkage: effects of boundary conditions and filling technique of resin composite restorations. J Dent 2004; 32(6):459-470.

5. Giachetti L, Scaminaci Russo D, Bambi C, Grandini R. A review of polymerization shrinkage stress: current techniques for posterior direct resin restorations. J Contemp Dent Pract 2006;7(4):79-88

6. Belvedere PC. Contemporary posterior direct composites using state-of-the-art techniques. Dent Clin North Am 2001;45(1):49-70

7. Davidson CL, Feilzer AJ. Polymerization shrinkage and polymerization shrinkage stress in polymer-based restoratives. J Dent 1997; 25(6):435-440.

8. Tantbirojn D, Versluis A, Pintado M, DeLong R, Douglas $\mathrm{W}$. Tooth deformation patterns in molars after composite restoration. Dent Mater 2004;20(6):535-542.

9. Feilzer AJ, De Gee AJ, Davidson CL. Setting stress in composite resin in relation to configuration of the restoration. J Dent Res 1987; 66(11):1636-1639.

10. Versluis A, Tantbirojn D, Douglas W. Do dental composite always shrink toward the light? J Dent Res 1998;77(6):1435-1445.

11. Campodonico C.; Tantbirojn D.; Olin P. S.; Versluis A: cuspal deflection and depth of cure in resin based composite restorations filled by using bulk, incremental and transillumination techniques. JADA 2011; 142(10):1176-1182.

12. Alshaafi, M.M., Haenel, T., Sullivan, B., Labrie, D., Alqahtani, M.Q. and Price, R.B., (2015). 'Effect of a broad- spectrum LED curing light on the Knoop microhardness of four posterior resin based composites at 2, 4 and 6-mm depths'. Journal of Dentistry, 45:14-18.

13. Schneider LFJ, Consani S, Sinhoreti MA, Sobrinho LC \& Milan FM Temperature change and hardness with different resin composites and photo-activation methods. Operative Dentistry (2005) 30(4) 516-521.

14. Vandewalle KS, Roberts HW, Tiba A \& Charlton DG Thermal emission and curing efficiency of LED and halogen curing lights Operative Dentistry(2005) 30(2) 257-264.

15. Silva, E.M.D., Almeida, G.S., Poskus, L.T. and GuimarÃ£es, J.G.A.,. 'Relationship between the degree of conversion, solubility and salivary sorption of a hybrid and a nanofilled resin composite'. Journal of Applied Oral Science, (2008)16 (2):161-166.

16. Leprince, J.G., Leveque, P., Nysten, B., Gallez, B., Devaux, J. and Leloup, G.,. 'New insight into the "depth of cure" ofdimethacrylate-based dental composites'. Dental Materials, (2012) 28 (5):512-520.

17. Abed, Y.A., Sabry, H.A. and Alrobeigy, N.A., 'Degree of conversion and surface hardness of bulk-fill composite versus incremental-fill composite'. Tanta Dental Journal, (2015). 12 (2):71-80.

18. Poggio, C., Lombardini, M., Gaviati, S. and Chiesa, M., 'Evaluation of Vickers hardness and depth of cure of six composite resins photo-activated with different polymerization modes'. Journal of Conservative Dentistry, (2012). 15 (3):237.

19. Alshali, R.Z., Salim, N.A., Satterthwaite, J.D. and Silikas, N., 'Post-irradiation hardness development, chemical softening, and thermal stability of bulk-fill and conventional resin-composites'. Journal of Dentistry, (2015). 43 (2):209-218.

20. Lazarchik DA, Hammond BD, Sikes CL, Looney SW, Rueggeberg FA. Hardness comparison of bulk-filled/transtooth and incrementalfilled/occlusally irradiated composite resins. J Prosthet Dent 2007; 98(2):129-140.

21. Amaral CM, de Castro AKBB, Pimenta LAF, Ambrosano GMB. Influence of resin composite polymerization techniques on micro -leakage and microhardness. Quintessence Int 2002;33(9):685-689. 